\title{
Philosophiques
}

\section{Céline Spector, Éloges de l'injustice. Philosophie face à la déraison, Paris, Seuil, 2016, 240 pages}

\section{Ludmilla Lorrain}

Volume 45, numéro 1, printemps 2018

URI : https://id.erudit.org/iderudit/1048633ar

DOI : https://doi.org/10.7202/1048633ar

Aller au sommaire du numéro

Éditeur(s)

Société de philosophie du Québec

ISSN

0316-2923 (imprimé)

1492-1391 (numérique)

Découvrir la revue

Citer ce compte rendu

Lorrain, L. (2018). Compte rendu de [Céline Spector, Éloges de l'injustice.

Philosophie face à la déraison, Paris, Seuil, 2016, 240 pages]. Philosophiques,

45(1), 316-321. https://doi.org/10.7202/1048633ar d'utilisation que vous pouvez consulter en ligne.

https://apropos.erudit.org/fr/usagers/politique-dutilisation/ 
chrétien que la question est abordée, et c'est chez Bonaventure que l'auteur retrouve les outils conceptuels qui conviennent à l'expérience qu'il cherche à décrire: la «nihilitét5 ${ }^{45}$ et l'humilité.

L'ouvrage se conclut par un texte écrit par Garth Green ${ }^{46}$, qui laisse l'ouvrage en héritage aux lecteurs, "car la signification d'un événement et celle d'un texte n'appartiennent qu'à l'avenir auquel ils donnent lieu ${ }^{47}$ ».

Religion et vérité est d'abord un ouvrage courageux, en ce qu'il tente de montrer, à travers un spectre très large d'approches, l'« incontournabilité» (pour le dire avec Heidegger) de la vérité religieuse. C'est pourquoi il s'agit d'un livre susceptible d'intéresser tant les spécialistes de philosophie de la religion, que les lecteurs prêts à se questionner sur le sens et le fondement philosophique de leur croyance ou non-croyance. Certaines positions exprimées là ne manqueront pas de contrarier certains, mais même les thèses les plus audacieuses laissent transparaître en filigrane une réflexion philosophique. Finalement, on remarque que l'ouvrage est dédié «à la mémoire des victimes des attentats du 7 janvier et du I3 novembre 20I5»: puisqu'il s'agit des actes d'un colloque ayant eu lieu au Québec, rien n'aurait empêché de dédier (aussi) cette belle publication à la mémoire des victimes de l'attentat du 29 janvier 20I7 à la Grand Mosquée de Québec. Peut-être le délai éditorial ne l'a-t-il pas permis?

GIULIO MELLANA

Université Laval, Québec Sorbonne Université, Paris

\section{Céline Spector, Éloges de l'injustice. Philosophie face à la déraison, Paris, Seuil, 2016, 240 pages.}

«Avions-nous oublié le mal?»: cette question — reprise du titre de l'ouvrage de Jean-Pierre Dupuy — est au cœur du livre de Céline Spector, Éloges de l'injustice, la philosophie face à la déraison. Ainsi, si le livre de JeanPierre Dupuy, paru en septembre 2002, interrogeait l'incapacité de la philosophie politique contemporaine à comprendre les attentats du I I septembre 200I, l'ouvrage de C. Spector s'ouvre — et se clôt — sur la difficulté du discours philosophique à produire une analyse convaincante des attentats qui, particulièrement depuis 2015, ont touché la France. On pourrait croire de la sorte que l'ouvrage répond à l'urgence de l'événement - avec les risques que comporte cette forme d'écriture. Mais il n'en est rien. En effet,

45. Ibid., p. 262.

46. Garth Green, « Religion et vérité: le passé et le futur d'une conjonction », Jean Grondin et Garth Green, dir., Religion et vérité. La philosophie de la religion à l'âge séculier, p. 26727 I.

47. Ibid., p. 269. 
le constat d'un échec du discours philosophique à l'endroit du terrorisme ne donne lieu qu'à une analyse très brève de ces événements - qui n'est pas, selon nous, la partie la plus importante de l'ouvrage. En vérité, ce constat engage un travail d'histoire de la philosophie qui entend comprendre ce qu'a perdu la pensée politique contemporaine, qui la rend incapable de saisir le réel qu'elle se propose pourtant d'éclairer.

Ainsi le véritable objet du livre est-il l'étude des éloges de l'injustice qu'ont produit les philosophes eux-mêmes. C. Spector se propose ainsi de suivre ces "personnages conceptuels » qui, comme le soulignait Deleuze et Guattari - auxquels elle emprunte l'expression -, ne sont pas des "personnification[s] abstraite[s] ${ }^{1}$ ", mais des "êtres fictifs figurés en chair en $O s^{2}$ ». L'ouvrage s'organise donc autour de ces figures, les cinq premiers chapitres étudiant leurs incarnations dans la pensée classique - les sophistes Calliclès et Thrasymaque de Platon au chapitre I, le «foole» de Hobbes au second chapitre, le «raisonneur violent» de Diderot au chapitre III, «l'homme indépendant» de Rousseau, et le «fripon intelligent» de Hume au chapitre IV; enfin, le libertin de Sade au chapitre V. Le dernier chapitre, quant à lui, s'attache à montrer, fort des analyses des chapitres précédents, la manière dont la philosophie politique contemporaine de tradition anglosaxonne - telle qu'elle s'est développée dans le sillage de Rawls et de sa Théorie de la justice - a "tourné le dos à l'histoire ", oubliant du même coup la figure de l'Insensé. Or cet oubli est dramatique, car l'Insensé pose une difficulté de taille à l'entreprise philosophique: comment convaincre celui qui sacrifie la raison au désir égoïste de la supériorité du juste? Deux projets se croisent donc dans cet ouvrage. Le premier, qui s'occupe essentiellement d'histoire de la philosophie, explique son parcours: une galerie des figures de l'injustice, de Platon à Sade. Le second, plus souterrain, mais aussi plus polémique, lui donne son but - et probablement son énergie: expliquer la cécité du discours philosophique dominant. Ces deux projets sont solidaires: comprendre l'échec de la philosophie politique contemporaine implique de saisir ce que les anciens savaient que nous aurions perdu.

La philosophie politique a-t-elle donc oublié le mal? C'est ce que lui reproche C. Spector. Comme le souligne le chapitre VI, si celle-ci convoque bien une figure de l'injuste, c'est sous une forme profondément transformée, celle du «resquilleur». Figure médiocre du profiteur, le resquilleur est celui qui veut bénéficier des biens produits par l'action collective sans fournir sa part à l'effort commun. Or, si le free rider n'est qu'un «égoïste borné qui n'entend pas se soumettre aux termes de la coopération s'il n'y voit pas son

1. Céline Spector, Éloges de l'injustice. Philosophie face à la déraison, Paris, Seuil, 2016, p. 18 .

2. Ibid., p. 19.
3. Ibid., p. 26. 
avantage immédiat ${ }^{4}$ », il est toujours possible de lui signifier ses erreurs de calcul. Et de lui montrer que la coopération est en vérité à son avantage. Sous les traits du free rider, l'intégration de l'Insensé à la théorie du choix rationnel produit ainsi une forme de lissage, réduisant la question de l'injustice à la nécessité d'empêcher la prolifération de ce type de comportements dans la société - soit en convaincant l'injuste de son erreur, soit en l'obligeant à la coopération, par le jeu mécanique des institutions.

En ce sens, C. Spector semble surtout regretter l'«infiltration» de la logique économique dans le domaine politique. Car elle conduit à oublier que les hommes ne sont pas que des êtres de raison, mais aussi - et le plus souvent - des êtres passionnés. Prendre pour paradigme le choix rationnel - ou la figure de l'homo oeconomicus ne cherchant qu'à maximiser son utilité - équivaut à rater la dimension passionnelle des comportements humains. Ce faisant, C. Spector souligne qu'en intégrant l'Injuste à leur fonctionnement, les "théories récentes de la justice ${ }^{5}$ " refusent de faire l'épreuve de leur mise en danger de l'extérieur. S'il est probable que la vingtaine de pages consacrées à cette tradition dans le chapitre VI n'en rende ni la richesse ni la diversité, ce choix nous dit quelque chose du projet de l'auteure. C'est que l'enjeu, nous semble-t-il, n'est pas tant d'entrer en discussion avec l'œuvre des auteurs et autrices de cette tradition, mais plutôt de questionner leur manière de faire de la philosophie.

L'histoire de la philosophie proposée par C. Spector doit donc montrer comment les philosophes qui figuraient dans leurs écrits l'Insensé acceptaient de la sorte de mettre leur édifice en danger. Car l'Insensé pose une question majeure à la philosophie morale et politique: comment justifier la "règle de réciprocité » qui enjoint à chacun de ne pas faire à autrui ce qu'il ne voudrait pas qu'on lui fasse. La figure de l'Injuste permet d'abord d'évaluer la force des résistances que rencontre cette affirmation. Ainsi, si les sophistes de Platon inaugurent la galerie des portraits, les traits majeurs de l'Insensé ne changent que très peu. L'Injuste apparaît comme celui à qui la rationalité du juste ne parle pas. Il n'entend pas voir ses projets limités par les exigences de justice. Il ne reconnaît pas la «règle de réciprocité ». Pourtant, son discours n'est pas irrationnel. Il donne au contraire toute sa force au conflit qui oppose la justice à l'intérêt. Loin d'être pure folie, la question qu'il pose au philosophe est essentielle: comment justifier, en pratique, que l'individu abandonne ce qui lui est profitable au nom de l'avantage commun? C. Spector montre combien, face à cette question, le philosophe semble bien embêté, lui qui, le plus souvent, se voit contraint de faire appel à un au-delà du logos pour convaincre, in extremis, ses adversaires. Ainsi, comme le rappelle le premier chapitre, Socrate ne parvenant pas à convaincre Calliclès de la rationalité du juste finit par faire appel à la vie après la mort.

4. Ibid., p. 85 .

5. Ibid., p. 25 . 
Se dégage donc de ces portraits une même figure de l'Injuste, dont le discours, que l'on pourrait dire "réaliste", voire cynique, nous enjoint de reconnaître que la justice n'est autre chose que convention, et qu'il est toujours plus conforme à la nature de suivre ses désirs que l'avantage commun. À cet égard, la figure du libertin de Sade pousse l'antinomie du juste et de l'intérêt à son paroxysme. Les pages que C. Spector consacre à cet auteur sont d'ailleurs passionnantes, celles-ci y montrant comment Sade retourne, point par point, la théorie morale et politique de Rousseau. Ainsi, si l'argument maître de Sade consiste à dire que la nature nous parle encore à l'état social, ce qu'elle nous dit selon lui est bien différent du discours tenu par la conscience chez Rousseau. Pour Sade, en effet, «suivre la nature suppose de comprendre que la seule mesure du vivant est celle de l'intensité des sensations physiques ${ }^{6} »$. De la même manière, si Sade fait sienne l'idée d'une société régie par un "pacte de dupes", ce constat n'est plus l'occasion de dénoncer un homme dénaturé, mais de justifier la méchanceté des pauvres. Ainsi la Dubois explique-t-elle à Justine que «la dureté des richesses légitime la mauvaise conduite des pauvres ${ }^{7}$ ». Chez Sade, et particulièrement dans Justine ou les malheurs de la vertu, la pitié est sans cesse humiliée. Les intermèdes, qui ponctuent chaque chapitre, sont en outre l'occasion de redoubler la force de cette difficulté, en montrant comment la littérature l'a mise en scène. Ainsi l'Intermède 5, dans lequel C. Spector étudie la figure de Dom Juan, met-il en évidence la nécessité d'un principe supérieur au logos pour, sinon convaincre l'immoraliste, au moins le punir: Dom Juan n'est-il pas puni par Dieu lui-même? Pourtant, cet appel à l'immortalité semble constituer une base bien fragile pour la justice. Et l'on peut ainsi se demander si, en dehors de la fiction, les Richard III, loin d'échouer comme dans la pièce de Shakespeare que C. Spector convoque dans l'Intermède 4 , ne sont pas plutôt ceux qui, le plus souvent, s'en sortent.

Suivant les philosophes qu'elle étudie, C. Spector parvient parfaitement à montrer la force du réaliste cynique, qui dispose d'arguments extrêmement puissants, exerçant sans conteste un fort pouvoir d'attraction. Et c'est finalement chez Rousseau, dont elle est par ailleurs spécialiste, que l'auteure semble trouver une résolution possible de l'aporie du juste et de l'intérêt. Celle-ci suppose que l'on renonce à convaincre l'Injuste. Aussi le «coup de force» de Rousseau est-il d'avoir compris que la seule manière de s'adresser à l'Insensé est de "jouer de l'appel à la sensibilitée ». C'est ce que met en scène la «Profession de foi du Vicaire savoyard» de l'Émile, qui s'adresse d'abord au cœur, avant ou au-delà de la raison. De la sorte, Rousseau parvient à dépasser, semble-t-il, l'aporie, en donnant «à la question

\footnotetext{
6. Ibid., p. 176 .

7. Ibid., p. 179 .

8. Ibid., p. I 59 .
} 
classique de la rationalité du juste une forme nouvelle ${ }^{9}$. Il faut, en effet, ancrer la justice dans l'intérêt, en posant la bonté originelle de l'homme, dont dérivent les principes premiers que sont l'amour de soi et la pitié. Si, comme le suggère Rousseau, la voix de la conscience seule nous donne accès au bien, la supériorité du juste doit être découverte par autre chose que la raison. Suivant Rousseau, C. Spector dégage ainsi une thèse forte: "La rationalité du juste ne se démontre pas. Elle suppose l'épiphanie d'une voix intérieure-extérieure, seule capable de contrer la force des passions ${ }^{10}$.»In fine, l'adhésion à la vertu de justice ne dépend donc pas d'une conviction, mais d'une conversion du cœur.

Néanmoins, l'appel au cœur adressé à l'immoraliste ne semble pas répondre au problème posé par la figure du free rider. En effet, telle que décrite par C. Spector, cette figure ne prône pas l'immoralité radicale. Le passager clandestin est même sûrement convaincu de l'utilité du juste. S'autorisant seulement des écarts, il traduit plutôt une pensée prosaïque: on peut bien faire des exceptions. "Il est, nous dit C. Spector, l'individu qui, sans méchanceté particulière, sans agressivité déplacée, détache son intérêt de l'intérêt commun ${ }^{11}$.» Or ce personnage aussi a une histoire. Contre la tradition anglo-saxonne qui le fait remontrer au foole de Hobbes - C. Spector montrant bien, au chapitre II, les raisons pour lesquelles cette lecture est insuffisante -, l'ancêtre du passager clandestin n'est autre que le Neveu de Rameau, mis en scène par Diderot dans son roman du même nom. Cette généalogie alternative du free rider permet de dresser le portrait d'un individu dont le rapport au juste et à l'injuste est somme toute banal: "dans un monde corrompu, [l'injustice] seule est rationnelle, c'est-à-dire payante ${ }^{12}{ }_{\text {». }}$.

Il nous semble en outre que cette histoire du free rider permet à C. Spector de mettre en évidence deux choses essentielles. D'une part, cette généalogie alternative permet de montrer que prendre en considération ce personnage conceptuel n'annule pas la nécessité de penser la figure du «raisonneur violent» - celui qui, intransigeant, refuse violemment l'ordre social établi. L'injustice a donc plusieurs visages, et la tâche du philosophe est de "cartographier leur diversité ${ }^{13}$ ». Plus encore, cette généalogie permet de montrer que ce personnage lui-même ne fonctionne pas seul: on ne pense le passager clandestin qu'une fois la question de l'injustice radicale réglée. Ainsi, il ne s'agit aucunement de minimiser les difficultés que posent la figure $\mathrm{du}$ free rider - et, comme le souligne l'auteure, Rousseau ne s'y est pas trompé, prenant très au sérieux la menace persistante du retour de l'intérêt particulier contre l'intérêt commun, de l'homme contre le citoyen — mais

\footnotetext{
9. Ibid., p. I 59 .

10. Ibid., p. I 54 .

11. Ibid., p. I39.

12. Ibid., p. I 6 .

13. Ibid., p. 227.
} 
plutôt, d'en contester l'hégémonie. Se dégagent donc de l'ouvrage deux grandes figures de l'injustice qui, selon la généalogie proposée par C. Spector, ont cohabité chez les philosophes jusqu'à la fin du XvIII siècle. L'Insensé, qui, au nom d'une nature impérieuse, rejette toute forme de justice incarné avec éclat sous la plume de Sade. L'injuste ordinaire, "profiteur » ou "parasite », qui n'a pas le panache de l'Injuste, mais dont le potentiel de nuisance, pour cette raison même, est tout aussi puissant.

Ainsi, l'idée centrale de cet essai semble bien être que la philosophie ne saurait se contenter de confronter cette dernière figure. Confrontation trop facile, puisque le philosophe et le passager clandestin ont la rationalité en partage. Au-delà de la question du mal, c'est bien à une réflexion sur les limites du discours philosophique que cet ouvrage nous invite. Car la confrontation du philosophe à l'Insensé amène nécessairement à se poser la question du destinataire du discours philosophique, ainsi que celle de sa portée. Si les arguments du logos ne sont d'aucune valeur pour celui qui n'en reconnaît pas la primauté, ne doit-on pas reconnaître que tout l'effort du philosophe se réduit à prêcher des convaincus? Quel est, dès lors, l'enjeu de cette confrontation? Pour C. Spector, il semble qu'incarner l'altérité doive permettre de «mesurer l'ampleur des résistances au discours sur la rationalité du juste ${ }^{14}$ » pour les comprendre, mais aussi tenter de les anticiper. Ainsi, si C. Spector travaille la question, classique s'il en est, du mal moral, il nous semble qu'elle engage, dans le même temps, une réflexion nécessaire sur ce que doit être la philosophie politique, dans sa méthode comme dans ses objets.

LUDMILLA LORRAIN Université Paris 1, Panthéon-Sorbonne

\section{Vincent Citot, dir., Problèmes épistémologiques en histoire de la philosophie, Montréal, Liber, 2017, 398 pages.}

Pourquoi faire de l'histoire de la philosophie? Et comment faire de l'histoire de la philosophie? Telles sont les questions fondamentales qui se posent dans le champ de l'épistémologie de l'histoire de la philosophie. Le premier mérite de l'ouvrage dirigé par Vincent Citot est de placer ces questions au centre du débat. L'ouvrage comble ainsi un vide notable. Il y a en effet quelque chose d'étonnant dans le fait que ces questions soient assez rarement abordées dans les premières années du cursus universitaire alors que l'histoire de la philosophie y occupe une place importante. Tandis que ces questions font la plupart du temps l'objet d'un enseignement spécifique chez les historiens et les sociologues, un étudiant en philosophie peut, sans doute 